\title{
Una nueva metodología para la evaluación del riesgo potencial de un episodio de precipitaciones extremas en un entorno operativo: aplicaciones
}

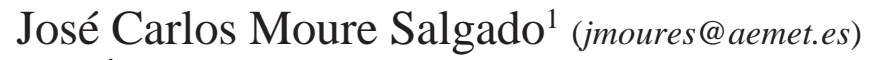

José Ángel Abreu Castiñeira² (angel.abreu@gmx.net)

${ }^{1}$ AEMET / Delegación Territorial en Galicia

${ }^{2}$ Swiss Federal Institute Aquatic Science Technology, Dübendorf, Suiza

\begin{abstract}
RESUMEN
En este trabajo se expone una metodología que, mediante un enfoque analítico y utilizando el modelo de alta resolución del ECMWF, estratifica la atmósfera en niveles o estados que permiten evaluar cuantitativamente, en un entorno operativo, el riesgo potencial de un episodio meteorológico asociado a intensidades de precipitación de alto impacto, es decir, aquellas que pueden desviarse significativamente de la climatología.

Una importante aplicación de dicha metodología consiste en que puede utilizarse para investigar de forma objetiva el papel humano en los avisos de precipitación y su capacidad para proporcionar valor añadido a la salida numérica de los modelos de predicción meteorológica. La percepción asimétrica que se tiene de los fallos y las falsas alarmas (una falsa alarma no suele causar víctimas) es inmediatamente absorbida cuando se formula la calidad de los avisos en términos de la razón coste/pérdida (valor económico). De esta forma se ha podido cuantificar su utilidad al considerar cuándo los pronósticos son beneficiosos, neutros o perjudiciales para los usuarios en función de sus necesidades y, a partir de aquí, cuándo las medidas llevadas a cabo por autoridades y particulares no serían recomendables y cuándo verdaderamente sí precisarían actuación en relación a un pronóstico base no hábil como es la climatología como referencia. En particular, se evalúa el rendimiento de los avisos de precipitación emitidos por AEMET en Galicia.
\end{abstract}

PALABRAS CLAVE: integral vertical de la divergencia de la humedad; valor añadido; aviso meteorológico; verificación; razón coste/pérdida.

\section{INTRODUCCIÓN}

En la predicción operativa se combinan una variedad de estrategias que integran modelos, teoría y observaciones en el proceso de análisis que, junto con el valor añadido del predictor, contribuyen a tomar una decisión final sobre un pronóstico determinado. No obstante, el papel de los seres humanos en el proceso de predicción no ha sido sometido a un minucioso y completo estudio (discutido por Doswell, 2004) y tampoco se dispone de ningún estudio contrastado y sometido a rigurosa verificación sobre la eficacia de una amplia variedad de variables utilizadas en la predicción operativa para determinar la probabilidad de un tiempo adverso asociado a precipitaciones extremas (discutido por Doswell y SCHULTz, 2006). 
Un sistema de pronóstico que utilice un gran volumen de información dificulta el juicio (combinación de intuición y análisis) y la toma de decisiones (Doswell, 2004; SAREwITZ et al., 2000). Por el contrario, una metodología que utilizase umbrales sometidos a verificación rigurosa evitaría la evaluación subjetiva de la predicción, filtrando la información tangencial, centrando la discusión y limitando el número de cifras, convirtiéndose en un asunto clave a la hora de tomar una decisión correcta sobre un pronóstico determinado.

Con este propósito se expone una metodología (de aquí en adelante, se utilizará VM para referirse a ella) que discretiza la atmósfera en estados o niveles medibles utilizando un parámetro que depende de dos variables y que permite evaluar de forma objetiva el riesgo potencial de un episodio de precipitaciones extremas en un entorno operativo. Aunque el estudio se centra en Galicia, la metodología desarrollada tiene carácter completamente general y podrá aplicarse, sin restricciones, a sistemas asociados al paso de sistemas a escala sinóptica o de mesoescala en latitudes medias.

\section{MECANISMOS DETERMINANTES EN LAS PRECIPITACIONES EXTREMAS}

\subsection{Un modelo de precipitación convectiva}

Un punto de partida apropiado para investigar mecanismos determinantes involucrados en altas tasas de precipitación, aparte de observaciones remotas (por ejemplo, radar Doppler) o in situ dentro de las nubes, podría encontrarse en un modelo de precipitación convectiva que haya mejorado nuestra compresión cualitativa de la convección sin entrar en los complejos procesos asociados a la microfísica de las nubes. EMANUEL (1986) desarrolló un modelo lineal de precipitación convectiva en donde se establecen los procesos dinámicos básicos (como la aparición de corrientes inclinadas y capas de cizalladura) que se derivan de la caída de la precipitación. A partir de la ecuación de la dinámica y del balance de vapor de agua en dicho modelo puede demostrarse que la variación de la energía cinética por unidad de masa en la corriente de aire (directamente relacionada con la tasa o intensidad de precipitación) puede expresarse de la siguiente manera:

$$
K-K_{0}=\int_{z} \frac{\rho}{\rho_{0}} g d z-\frac{g}{\rho_{0}} \int_{t}\left\{\int_{z}-(\vec{v} \cdot \nabla q) \frac{d p}{g}\right\} d t+\text { otros términos }
$$

El primer término del segundo miembro representa la energía potencial convectiva disponible (Convective Available Potential Energy, CAPE ) mientras que la cantidad entre corchetes se denomina integral vertical de la divergencia de humedad (Vertically Integrated Moisture Divergence, VIMD), que integrada en el tiempo la expresaremos VIMD ${ }^{t}$.

La precipitación total $P$ observada en cualquier punto está determinada por la tasa de precipitación observada $\sigma$ y por la duración $D$, de modo que $P=\sigma \cdot d$. Truncando la ecuación [1] a partir de los dos primeros términos, vamos a investigar en qué medida estas dos variables, una de naturaleza dinámica VIMD ${ }^{t}$, y otra de naturaleza termodinámica, que pueden ser la $C A P E$ o, alternativamente, el lifted index ( $L I$ ), juegan un papel importante como factores moduladores sobre $\sigma$ y su capacidad para definir cuantitativamente distintos estados de la atmósfera en relación con los episodios asociados a precipitaciones extremas. Todas las variables mencionadas se formulan como cantidades integrales y tienen significado físico de modo que pueden ser aplicadas e interpretadas a nivel global.

\subsection{Definición matemática de la VIMD según el ECMWF}

Para obtener los valores numéricos de las variables $\left(V I M D^{t}, C A P E / L I\right)$ se necesita una red tridimensional de la atmósfera con observaciones directas de las magnitudes físicas involucradas. Esto, obviamente, no es posible, por lo que es necesario abordar la cuestión a partir de pronósticos de modelos de predicción meteorológica. En este sentido, el modelo de alta resolución (HRES) del Centro Europeo de Predicción a Plazo 
Medio (ECMWF) proporciona la integral vertical de la divergencia de humedad y cuya definición matemática es la siguiente (según el área de modelización numérica del ECMWF, comunicación personal):

$$
V I M D \equiv-\int_{s f c}^{\infty}\left(\vec{v} \cdot \nabla q_{T}\right) \frac{d p}{g}\left(\frac{\mathrm{kg}}{\mathrm{m}^{2} \cdot \mathrm{s}}\right)
$$

Representa la integral vertical, desde la superficie hasta el límite superior de la atmósfera, de la advección 3-D de la razón de mezcla de especies húmedas (vapor de agua, agua líquida e hielo). La ecuación es integrada en el tiempo para obtener el diagnóstico final en cada punto de grid del modelo,

$$
\overline{V^{\prime} I M D^{t}}=\Delta t \text { VIMD }
$$

donde $\overline{V I M D^{t}}$ es una aproximación discreta a

$$
\int_{0}^{t}\left\{\int_{s f c}^{\infty} \vec{v} \cdot \nabla q_{T} \frac{d p}{g}\right\} d t\left(\frac{\mathrm{kg}}{\mathrm{m}^{2}}\right)
$$

La $\overline{V I M D^{t}}$ computa solo el efecto de la dinámica, es decir, los terminos procedentes de la física (fundamentalmente procesos en la capa límite, orografía, tipo de suelo, vegetación, turbulencia, etc.) no se incluyen. Esto es importante ya que: i) los mecanismos que involucran las precipitaciones extremas en la península ibérica y, en general, en latitudes medias tienen su fuente principal en procesos dinámicos, es decir, procesos atmosféricos que implican el movimiento del aire, tales como advección, fuerzas de gradiente de presión así como la expansión y compresión adiabática; ii) los modelos espectrales, tales como el IFS (Integrated Forecast System) del ECMWF, tienden a generar una mejor representación de los campos globales y de la dinámica que los modelos de área limitada (COMET, 2010); iii) al no incluir procesos que dependen fuertemente de la resolución horizontal, los cambios en la resolución espacial del modelo (el modelo del ECMWF sufrió una modificación en marzo de 2016 y la longitud de rejilla pasó de 16 a 9 km) tienen un efecto limitado sobre la interpretación cuantitativa de cuando es integrada en el tiempo.

La segunda variable combinada de interés puede ser la CAPE o el LI. El modelo del ECMWF calcula la CAPE de la parcela más inestable de los $350 \mathrm{hPa}$ más bajos de la atmósfera y el LI puede obtenerse a partir de las variables del modelo.

\section{METODOLOGÍA}

\subsection{Observaciones de precipitación y rayos}

Los datos de precipitación observada proceden de la red de estaciones automáticas de AEMET, confederaciones hidrográficas y de MeteoGalicia (en total 252 estaciones) y los rayos observados de la red de detección de rayos de AEMET y de MeteoGalicia lo que permite también identificar las tormentas. En todo el periodo se supone que los errores instrumentales son pequeños frente a los errores de los pronósticos, con datos que han sido sometidos a un control de calidad. La red de estaciones automáticas y de rayos en Galicia se considera bien espaciada geográficamente en relación a las predicciones por lo que los errores de muestreo también se consideran pequeños. La ausencia de datos durante el periodo de tiempo analizado ha sido puntual y puede suponerse que se ha producido al azar, por lo que la verificación de los pronósticos no debería verse afectada.

\subsection{Estados de la atmósfera: obtención de los niveles de riesgo en función de $\sigma$}

Para definir cuantitativamente los diferentes estados de la atmósfera se utilizó el HRES del ECMWF (resolución $0,125^{\circ} \times 0,125^{\circ}$ ) que proporciona el pronóstico más preciso para un periodo determinado aunque tiene 
la limitación de no proporcionar una estimación de la incertidumbre asociada. Los datos de precipitación de la red de estaciones automáticas que alcanzaron, al menos, el nivel de aviso amarillo según el Plan Nacional de Predicción y Vigilancia de Fenómenos Meteorológicos adversos (Meteoalerta) para Galicia se recopilaron inicialmente desde noviembre de 2011 (fecha desde la que se dispone de la $\overline{V I M D^{t}}$ horaria [ $\Delta t=1$ hora] como campo de pronóstico del ECMWF) hasta diciembre de 2015, un periodo de tiempo suficiente para establecer una climatología fiable que incluya el rango completo de observaciones en Galicia. Los campos de pronóstico horarios desde $\mathrm{T}+0$ hasta $\mathrm{T}+14$ sobre Galicia $\left(46^{\circ} \mathrm{N}, 12^{\circ} \mathrm{W}, 40^{\circ} \mathrm{N}, 6^{\circ} \mathrm{W}\right)$ se utilizan de la siguiente forma:

i) En cada estación de la red en la que se alcanzó, al menos, el nivel de aviso amarillo observado se calculó el valor de $\sigma=\sigma\left(\overline{V I M D}^{t}, C A P E / L I\right)$, eligiendo un intervalo de integración $\Delta t=3$ horas para la $\overline{V I M D^{t}}$ en un entorno de $15 \mathrm{~km}$ alrededor de la estación.

Las razones de la integración de la $\overline{V I M D^{t}}$ en 3 horas responde a diversas causas: factores físicos relacionados con la iniciación de la convección que puede empezar hasta 3 horas antes de que las células convectivas alcancen el estado de madurez (PuCILLO, 2009) o el tiempo de crecimiento (entre 1 y 3 horas) de las partículas de precipitación (hielo, gotas de agua) en las capas estratiformes (nimbostratos) adyacentes a la región convectiva (Houze, 2014) justifican ciclos de evolución trihoraria. Asimismo, la $\overline{V I M D^{t}}$ puede ser un número positivo o negativo, por lo que periodos de integración más largos podrían dar lugar a contribuciones excesivas mientras que intervalos de integración más cortos podrían aumentar excesivamente la sensibilidad a las pequeñas diferencias que puedan surgir de los errores de pronóstico del modelo.

Por otra parte, la elección de un radio de $15 \mathrm{~km}$ alrededor de cada estación deriva de la velocidad de desplazamiento promedio de los frentes y perturbaciones en general y que se sitúa en torno a $30 \mathrm{~km} / \mathrm{hora}$ (Houze, 2014). Con un campo de pronóstico horario es necesario un radio de $15 \mathrm{~km}$ en torno a cada estación para que la señal que se desplaza sobre ella a esa velocidad con la perturbación o frente se encuentre en algún momento dentro de su radio de acción en un intervalo de 1 hora y pueda ser cuantificada.

ii) A continuación se definieron distintos estados de la atmósfera según los valores numéricos de $\sigma=\sigma\left(\overline{V I M D^{t}}, C A P E / L I\right)$ mediante un criterio objetivo de frecuencia de ocurrencia (probabilidad) de un evento adverso asociado a la precipitación de la siguiente forma (tanto para las precipitaciones acumuladas en 1 hora como en 12 horas):

— Definición: $\Re_{1}$ (alternativamente $\Re_{2}$ ) es el estado de la atmósfera en el cual la probabilidad de que se alcance, al menos, en una estación de una zona meteorológica de aviso el nivel de aviso amarillo de precipitación observada es igual o mayor del $70 \%$ (alternativamente $40 \%$ ). Si la probabilidad es inferior al $40 \%$ se definen los estados de la atmósfera $\Re_{3} \mathrm{o} \Re_{4}$, según se presenten fenómenos convectivos asociados a tormentas o no.

A partir de la definición anterior, se obtuvieron los estados de la atmósfera esquematizados en la figura 1 con los correspondientes rangos de valores de $\sigma$. Cada estado representa un nivel de riesgo $\Re_{i}$ de un evento de tiempo adverso asociado a la precipitación (tabla 1) según el plan Meteoalerta para Galicia. Se utiliza el LI como variable termodinámica ya que mostró el mejor comportamiento cuando se estudiaron comparativamente diferentes índices de inestabilidad. Estudios previos (Van Zomeren y Van Delden, 2005) apuntan en la misma dirección en la parte occidental de Europa. Cada dato de precipitación que ha alcanzado o superado el nivel de aviso amarillo en 1 y 12 horas está ahora etiquetado mediante un marcador meteorológico definido por $\sigma\left(\overline{V M D^{t}}, C A P E / L I\right)$.

La frecuencia esperada que se representa en la tabla 1 es la frecuencia de eventos observados respecto al total de ocasiones en las que el evento fue pronosticado y/u observado en cada estado de la atmósfera que ha sido definido y no la frecuencia climatológica de eventos de precipitación en los que se alcanza el nivel de aviso según el plan Meteoalerta para Galicia ya que esta probabilidad es muy pequeña. 


$$
\sigma=\sigma\left(\overline{V^{\prime} M D^{t}}, L I\right)=\left\{\begin{array}{ccc}
\mathfrak{R}_{4}: & \overline{V^{\prime} M D^{t}}>-18 & \text { LI } \geq 1 \\
\mathfrak{R}_{3}: & \overline{V I M D^{t}}>-12 & \text { LI }<1 \text { (tormentas) } \\
\mathfrak{R}_{2}: & \overline{V I M D^{t}} \leq-12 & L I<1 \text { (tormentas) } \\
\mathfrak{R}_{1}: & \overline{V I M D^{t}} \leq-30 & \text { LI cualquiera } \\
\mathfrak{R}_{0}: & \overline{V I M D^{t}} \leq-50 & \text { LI cualquiera (efemérides) }
\end{array}\right.
$$

Figura 1. Diagrama esquemático de los estados de la atmósfera definidos por los valores numéricos de $\sigma$ (mostrado para la precipitación acumulada en 12 horas).

\begin{tabular}{|l|c|c|c|}
\hline Nivel de riesgo & $\mathfrak{R}_{4} \mid \mathfrak{R}_{3}$ & $\mathfrak{R}_{2}$ & $\mathfrak{R}_{1} \mid \mathfrak{R}_{0}$ \\
\hline $\begin{array}{l}\text { Tastado atmósfera } \\
\text { el umbral Amarilio observado }\end{array}$ & $\leq 10 \%$ & $\geq 40 \%$ & $\geq 70 \%$ \\
\hline $\begin{array}{l}\text { Tasa de episodios que alcanzan } \\
\text { el umbral NARANJA/ROJo observado }\end{array}$ & $\leq 1 \%$ & $\leq 10 \%$ & $\geq 40 \%$ \\
\hline
\end{tabular}

Tabla 1. Frecuencia de ocurrencia de un episodio de precipitación extrema según las especificaciones de la figura 1 (mostrado para la precipitación acumulada en 12 horas).

\section{VERIFICACIÓN DE LOS AVISOS DE PRECIPITACIÓN}

\subsection{Valor económico de los avisos de precipitación}

La valoración de la calidad de los avisos de precipitación del sistema de predicción de AEMET para Galicia se realiza en términos de valor económico, con una perspectiva orientada al usuario. RICHARDSON (2000) demostró la siguiente relación:

$$
V=\frac{\operatorname{mín}(r, p)-F(1-p) r+H p(1-p)-p}{\operatorname{mín}(r, p)-p r}
$$

donde $V$ representa el valor económico de los pronósticos, denominado valor relativo, y expresa el beneficio general que un usuario puede esperar al tomar una larga secuencia de decisiones usando un determinado sistema de pronóstico; $p$ es la frecuencia esperada del evento y $r$ es la razón coste/pérdida $(C / L)$, siendo $0<r=C / L<1$; mín $(r, p)$ es el valor mínimo de $r$ o $p$ de manera que el usuario debe protegerse siempre si $r<p$ y no hacerlo nunca en caso contrario; $H$ es la probabilidad de detección y $F$ la probabilidad de falsa detección (JollifFe y STEPHENSON, 2012; WILKS, 2011). El máximo valor relativo de un sistema de predicción se obtiene haciendo $r=p$ en la ecuación [5] y viene dado por:

$$
V_{\text {máx }}=H-F
$$

En un sistema de ejes cartesianos, $V$ se representa en el eje de ordenadas. Si $V=1$ el sistema de pronóstico es perfecto y si $V=0$ no es mejor que la climatología. Los valores negativos de $V$ implican que el sistema de pronóstico es tan pobre que es más efectivo utilizar la climatología como método de pronóstico base no hábil. En el eje de abscisas se representa la razón $C / L$ en función de los intereses de los diferentes usuarios a la hora de tomar la decisión de proteger un activo frente al tiempo adverso pronosticado. 


\subsection{Principales características del procedimiento de verificación}

Para analizar si la metodología VM tiene potencial discriminatorio de los episodios de precipitaciones extremas, los avisos de precipitación de AEMET emitidos para Galicia en cada zona geográfica se distribuyeron en tres grupos $\left(\mathfrak{R}_{4} \mid \Re_{3}, \Re_{2}\right.$ y $\left.\Re_{1} \mid \Re_{0}\right)$ en función del estado de la atmósfera asociado. Para los avisos correspondientes a cada grupo se construyó la tabla de contingencia determinista de observaciones frente a predicciones y, a continuación, se dibujaron las curvas de variación de $V$ con $C / L$.

La verificación en términos de valor económico se realizó por separado para dos periodos, uno que abarca desde 2011 hasta 2015 (no mostrada) y otro desde 2016 hasta 2018 (figura 2). Este procedimiento aporta un conjunto de datos (periodo 2016-2018) completamente independientes (no contaminados) de los utilizados para el calibrado de la metodología VM (periodo 2011-2015). De este modo, si los resultados de la verificación de ambos periodos son equivalentes se puede descartar la habilidad artificial que tiene lugar cuando la calidad de una metodología se evalúa sobre el mismo conjunto de datos que fueron utilizados para su desarrollo. Se comprobó, asimismo, con un intervalo de confianza del $95 \%$ que los tamaños de las muestras elegidas en ambos periodos analizados eran lo suficientemente grandes para que las conclusiones obtenidas no pudieran explicarse por fluctuaciones aleatorias, es decir, por una casualidad del muestreo (THORNES y STEPHENSON, 2001).

Aunque los avisos de AEMET están formulados en términos de probabilidad $(>70 \%, 40 \%-70 \%$, $10 \%-40 \%$ ) de exceder un umbral durante el periodo de validez del pronóstico, la verificación se realizó únicamente para los que se les asignó una probabilidad entre el 40 y el $70 \%$ ya que el tamaño de muestra para los restantes es muy pequeña.

Se utilizó una metodología de verificación orientada a eventos (Moure, 2018) en cada una de las 16 zonas meteorológicas de aviso definidas por AEMET en el plan Meteoalerta para Galicia y, separadamente, para las acumulaciones de precipitación en 1 y 12 horas. Se considera que la superación del umbral de aviso en una estación pluviométrica dentro de cada zona meteorológica es suficiente para registrar la ocurrencia. Con objeto de definir $N$, el tamaño total de la muestra, se adoptó una discretización espacial y temporal de todo el periodo de verificación en todas las zonas meteorológicas (WILSON y GILES, 2013).

Para el conteo de negativos correctos solamente se incluyeron los episodios en los que se emitió un aviso de precipitación y/o se observó una precipitación que alcanzó el umbral de aviso en, al menos, una zona meteorológica según el plan Meteoalerta para Galicia. En la literatura científica (STEPHENSON et al., 2010) se sugiere que deben verificarse únicamente los episodios que cumplen con la condición de casos difíciles para evitar incluir muchas situaciones sencillas que introducirían un sesgo indeseado en la verificación. La verificación realizada cumple con dicha condición sin ambigüedad. En efecto, cualquier situación en la que se emite un aviso de precipitación, a priori, se supone un caso difícil independientemente de que dicha precipitación se alcance o no. Por otra parte, una precipitación observada que alcance el umbral de aviso, aunque este no haya sido emitido, sin duda es también un caso difícil.

\section{DISCUSIÓN DE RESULTADOS: VALOR ECONÓMICO DE LOS AVISOS DE PRECIPITACIÓN DE AEMET PARA GALICIA}

La similitud de las curvas de variación de $V$ con el ratio utilizando los avisos emitidos por AEMET para Galicia durante los periodos 2011-2015 y 2016-2018 puso de manifiesto la bondad de la metodología VM a la hora de discriminar la calidad de los avisos de precipitación en función del estado de la atmósfera.

Aunque se realiza una completa descripción del valor económico del sistema de predicción de AEMET para Galicia, en $\mathfrak{R}_{4} \mid \mathfrak{R}_{3}$ y en $\mathfrak{R}_{2}$ solo se realizó la verificación de avisos amarillos, no siendo necesaria la correspondiente a avisos naranjas/rojos y en $\Re_{1} \mid \Re_{0}$ únicamente la verificación de avisos naranjas y rojos, que se 


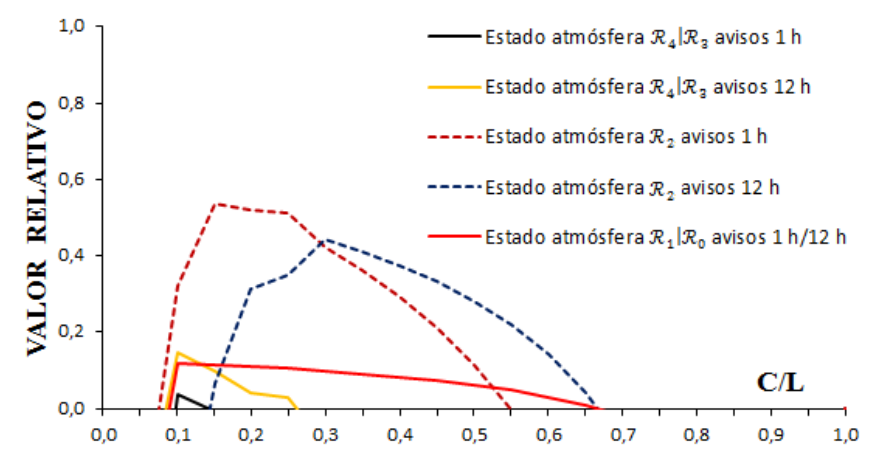

Figura 2.

Curvas de variación del valor relativo

(los valores negativos no se representan)

con el ratio $C / L$ obtenidas a partir de

los avisos de AEMET en Galicia

desde enero de 2016 hasta junio de 2018

en función del estado de la atmósfera.

llevó a cabo de forma conjunta para los avisos de 1 y 12 horas ya que el tamaño de la muestra de avisos naranjas y rojos de 1 hora es muy pequeña. Los aspectos más destacables mostrados en la figura 2 son los siguientes:

Cuando la atmósfera se encuentra en los estado de riesgo $\mathfrak{R}_{4} \mid \mathfrak{R}_{3}$, los avisos amarillos de 1 y 12 horas emitidos por AEMET para Galicia (líneas continuas negra y amarilla, respectivamente) no ayudan al usuario final a tomar decisiones adecuadas para, prácticamente, ningún rango de ratios $C / L$ de modo que las medidas de protección llevadas a cabo por autoridades políticas o los ciudadanos no son recomendables si tienen algún coste. Es más conveniente utilizar la climatología considerando que no se produce nunca un evento adverso como método de pronóstico. Señalar que más de la mitad de los avisos de precipitación de nivel amarillo emitidos por AEMET en Galicia se realizan bajo estas condiciones. La verificación de avisos de precipitación naranjas/rojos es innecesaria y se obtendrían conclusiones idénticas o más negativas, ya que $\mathfrak{R}_{4} \mid \mathfrak{R}_{3}$ constituye un estado de la atmósfera más desfavorable para el pronóstico de episodios aún más adversos.

Similares resultados se obtienen cuando la atmósfera se encuentra en el estado $\Re_{1} \mid \Re_{0}$ (línea continua roja); de nuevo el sistema de predicción de AEMET para Galicia exhibe muy escasa confianza. Los usuarios no pueden beneficiarse de los pronósticos en el rango de ratios donde $V$ es positivo ya que la precisión del valor relativo máximo, $V_{\text {máx }}$, con un intervalo de confianza del 95 \% (JOLLIFFE y STEPHENSON, 2012; WilKS, 2011) que se obtiene viene dada por:

$$
V_{\text {máx }}=(H-F) \pm \sqrt{(\Delta H)^{2}+(\Delta F)^{2}} \cong 0,1 \pm 0,1
$$

donde $\Delta H$ y $\Delta F$ representan, respectivamente, los errores estándar de $H$ y $F$. Por tanto, el intervalo de valores de $V_{\text {máx }}$ incluye el cero para todo el rango de ratios $C / L$ en que es positivo debido a la variabilidad aleatoria expresada mediante el error estándar y no puede garantizarse que los usuarios se puedan beneficiar de estas predicciones.

Este resultado es especialmente preocupante dado que los mayores beneficios para los usuarios de un sistema de predicción tiene lugar cuando logra una completa protección frente a grandes pérdidas, es decir, aquellas situaciones en las que el ratio $C / L$ tiende a ser muy pequeño y la inversión inicial está ampliamente justificada (por ejemplo, pérdidas de vidas) y estas situaciones, de producirse, cabrían esperarse en condiciones muy adversas en el nivel de riesgo $\Re_{1} \mid \Re_{0}$. Es innecesaria una verificación de avisos naranjas y rojos bajo un estado de la atmósfera en el nivel de riesgo $\mathfrak{R}_{2}$ ya que si bajo condiciones de la atmósfera que son muy favorables para su detección $\Re_{1} \mid \Re_{0}$ no se mejora la climatología como predicción de referencia, menos aún en $\Re_{2}$, un estado de la atmósfera que resulta menos favorable.

Solo cuando los avisos amarillos son emitidos en el estado de riesgo $\mathfrak{R}_{2}$ (líneas a trazos) las curvas de variación de $V$ con el ratio $C / L$, obtenidas a partir de los pronósticos, exhiben un significativo valor. Por ejemplo, los usuarios pueden lograr para los avisos de 1 h y un ratio $C / L \sim 0,15$ hasta un $56 \%$ del valor que 
obtendrían de un pronóstico determinista perfecto; esto se debe a que los avisos amarillos previstos por AEMET para Galicia tienen lugar justo en el estado de la atmósfera en donde existe una gran proporción de avisos amarillos observados y no a la habilidad del sistema de predicción.

Aunque reducir el número de falsas alarmas es un objetivo muy valioso, existe la percepción de que centrarse en la reducción de este indicador podría conducir a una mayor probabilidad de no detectar un evento extremo no deseado. Este trabajo desmonta esta percepción y pone de manifiesto que la calidad de las alertas amarillas puede ser un indicador apropiado del estado de salud de un sistema de predicción. En efecto, cuando los avisos fueron emitidos en el estado $\mathfrak{R}_{4} \mid \mathfrak{R}_{3}$ el porcentaje de falsas alarmas (amarillos previstos y no observados) superó el $80 \%$, mientras que cuando fueron emitidos en el estado $\mathfrak{R}_{1} \mid \mathfrak{R}_{0}$ el porcentaje de fallos (naranjas o rojos observados y no previstos) también superó el $80 \%$. Además, al evaluar la calidad de las alertas en términos de razón $C / L$, si el número de falsas alarmas es elevado las medidas de protección pueden encarecerse demasiado frente a las pérdidas potenciales, y no ser rentables.

Estos resultados siembran serias dudas de que exista un enfoque intuitivo humano, equivalente al que resulta de utilizar los valores de $\sigma$ de la figura 1, que sea capaz de discriminar eficazmente los episodios susceptibles de producir precipitaciones extremas. Los enfoques intuitivos eficaces a partir de la experiencia y el uso de modelos conceptuales no cuadran bien con el hecho de que las diferencias observadas en la precipitación son muy sensibles a los detalles de la circulación (ROEBBer y BOsART, 1998) y que los modelos conceptuales en los casos particulares tiene una precisión limitada (Doswell, 2004).

En este trabajo se han realizado una serie de suposiciones tanto en la metodología de verificación como en la inferencia estadística de las medidas. Así se supone que los datos son independientes, las 16 zonas meteorológicas tienen condiciones climáticas homogéneas y no existen cambios en la frecuencia esperada del evento pronosticado.

\section{CONCLUSIONES}

La confianza en la metodología VM es correspondientemente alta: los estados de la atmósfera definidos a través de $\sigma\left(\overline{V I M D^{t}}, L I\right)$ son determinantes para detectar aquellos episodios especialmente susceptibles de producir precipitaciones extremas y tomar una decisión de pronóstico adecuada.

La metodología VM tiene otras aplicaciones importantes ya que permite: i) evaluar la calidad de un sistema de predicción de forma robusta (utilizando avisos amarillos en $\Re_{4} \mid \Re_{3}$ y en $\Re_{2}$ ) sin depender de una pequeña cantidad de valores de precipitación extremos (los avisos naranjas o rojos en $\Re_{4} \mid \Re_{3}$ y en $\Re_{2}$ ) que impiden la verificación al no poder descartar que los resultados puedan deberse a la casualidad o que pueden tener una influencia indebida en la habilidad del sistema que se evalúa al enmascarar pronósticos para valores no tan extremos; ii) apreciar dónde se encuentran exactamente las principales deficiencias de los pronósticos y una mejor compresión de los procesos físicos involucrados en tales episodios. En particular, el sistema de avisos de AEMET para Galicia no es capaz de distinguir adecuadamente el riesgo potencial de los episodios de precipitación. Con frecuencia se valoran episodios con riesgo de precipitación extrema muy diferente como si fuesen iguales, no apreciándose ningún valor añadido ni mejoría a lo largo del tiempo y los potenciales usuarios apenas podrían beneficiarse económicamente de dicho sistema de predicción si lo usasen a largo plazo.

\section{AGRADECIMIENTOS}

Los autores quieren mostrar su agradecimiento a las entidades y personas siguientes: a la Delegación Territorial de AEMET en Galicia, en especial a J. P. García M., A. Garitagotia C., J. Hierro C. y J. M. Pascual R., al ECMWF, en especial a X. Abellán y a MeteoGalicia, organismo dependiente de la Consellería de Medio Ambiente de la Xunta de Galicia. 


\section{REFERENCIAS}

Cooperative Program for Operational Meteorology, Education and Training (COMET), 2010. Effective Use of numerical weather prediction (NWP) in the Forecast Process.

Doswell, C. A., III, 2004. Weather forecasting by humans: Heuristics and decision making. Weather and Forecasting, 19, 1115-1126.

Doswell III, C. A. y Schultz, D. M., 2006. On the use of indices and parameters in forecasting severe storms. Electronic Journal Severe Storms Meteorology, 1, 1-14.

EMANUEL, K. A., 1986. Some dynamical aspects of precipitating convection. Journal Atmospheric Science, 43, 2183-2198.

Emanuel, K. A., 1994. Atmospheric Convection. Oxford University Press: New York, NY.

Houze, R., 2014. Cloud Dynamics. Second Edition. Elsevier.

Jolliffe, I. T. y StePhEnSOn, D. B, 2012. Forecast Verification. Wiley. Second Edition.

Moure, J. C, 2018. Metodología de verificación de los avisos de precipitación. Delegación territorial de AEMET en Galicia. Documento interno. Sin publicar.

Pucillo, A. J., Giaiotti, D. B. y Stel, F., 2009: Ground wind convergence as a source of deep convection initiation. Atmospheric Research, 93, 437-445.

Richardson, D. S., 2000. Skill and economic value of the ECMWF ensemble prediction system. Quarterly Journal of the Royal Meteorological Society, 126, 649-667.

Roebber, P. J. y Bosart, L. F., 1998. The Sensitivity of Precipitation to Circulation Details. Part I: An Analysis of Regional Analogs. Monthly Weather Review, 126, 437-455.

Sarewitz, D., Pielke, R. A. y Byerly, R., 2000. Prediction, science, decision making and the future of Nature. Island Press.

SHARPE, M. 2016. A flexible approach to the objective verification of warnings. Meteorological Applications, $23,65-75$.

StePhenson, D. B., Jolliffe, I. y Ferro, C. A. T., 2010. White paper review on the verification of warnings. UK Met Office Technical Report No. 546. Met Office: Exeter, UK.

Thornes, J. E. y StePhenson, D. B., 2001. How to judge the quality and value of weather forecast products. Meteorological Applications, 8, 307-314.

VANZOMEREn, J. y VAN DeLDEN, A., 2005. Vertically moisture flux convergence as a predictor of thunderstorms. Atmospheric Research, 83, 435-445.

WILKS, D. S., 2011. Statistical Methods in the Atmospheric Sciences. Academic Press.

Wilson, L. J. y Giles, A., 2013. A new index for the verification of accuracy and timeliness of weather warnings. Meteorological Applications, 20, 206-216. 
